\title{
Cyto-histological aspects of haploid androgenesis when obtaining haploids/doubled haploids in rice (Oryza Sativa I.) anther culture in vitro
}

\author{
E. G. Savenko*, Zh. M. Mukhina, V. A. Glazyrina, and L. A. Shundrina \\ Federal Scientific Rice Centre, Krasnodar. Russia
}

\begin{abstract}
The aim of the study was to study the development of rice microspores in anther culture in vitro, study the structure of androclinic callus to identify cyto-embryological features of the formation of morphogenic structures in anther culture, obtain doubled rice haploids (Oryza sativa L.) and accelerate the development of valuable breeding material with desired properties. Within the framework of this study, the results of a cyto-histological study of rice haploid androgenesis in vitro were obtained, which indicate that it is induced under the influence of phytohormones from rice anther microspores at the mononuclear or early binuclear stage of development. The abnormal development of microspores on nutrient media with phytohormone 2,4-D was traced, in which nuclei, having lost their characteristic functions, acquired the ability to unlimited division and growth with the formation of microcallus. The morphological structure of calli was assessed. The main morphotypes of callus tissues and the pathways of morphogenesis, leading to the formation of androgenic structures, up to full-fledged regenerant plants, were identified. Homozygous androgenic lines based on $\mathrm{F}_{1}$ and $\mathrm{BC}_{1}$ - rice generations obtained in the course of hybridization and backcrossing between Chinese samples carrying blast resistance genes and Russian accessiond were rapidly developed.
\end{abstract}

\section{Introduction}

The production of doubled haploids (DH plants) is an important link in classical plant breeding and in fundamental research [1]. It occupies a leading place in breeding programs to accelerate the process of developing highly productive hybrids and varieties of agricultural plants [2]. Modern production requirements dictate the need to use innovative methods for obtaining haploids / doubled haploids (DH) to accelerate breeding programs to improve rice varieties (Herawati et al., 2010) [3]. The main breeding advantage of using haploids comes from the possibility of one-stage obtaining homozygotes, which allows you to quickly fix the morphophysiological parameters of adaptability and shorten the time for developing varieties adapted to the conditions of the region and numerous diseases, capable

* Corresponding author: avena5@rambler.ru 
of consistently forming high grain yields and meeting all the needs of the modern market. In haploids, each gene is represented by a single allele; recessive alleles of some genes appear along with dominant alleles of others. Genetic cleavage using haploids is less difficult and actually does not exceed the number of gamete classes, and a relatively small population is needed to isolate a certain combination of genes. Haploid technology is currently adapted in various plant breeding programs and is the most convenient approach for the rapid development of crops and the transfer of useful genes, chromosomal segments, and even complete chromosomes. DH populations are genetically similar to recombinant inbred lines; generated from a single seed source, they are used to map quantitative trait loci (QTLs). Despite some methodological advances, haploid biotechnology is still not widely used in practical breeding and fundamental research. [4, 5]. This is due to the low frequency of the release of embryoids, morphogenic calli, and regenerated plants in the isolated anthers and microspores culture in vitro (Lee and Lee, 2002) [6]. The stage of development of the sporogenous cell, favorable for anther inoculation, largely determines the success of the cultivation of isolated anthers of both cereals and representatives of other families.

Microspores and pollen grains respond to in vitro conditions only within the "morphogenic window" - a limited period of time during which certain external factors can "switch" the gametophytic path of pollen differentiation to the sporophytic one. Literary sources provide accurate information about the phase of development of anthers and microspores for some types of crops, but there is no consensus about the phase of development of microspores and pollen grains for the beginning of cultivation of anthers of cereals, including rice $[7,8]$.

It is believed that the period of induction of androgenesis in cereals is a late phase of microsporogenesis, while it is noted that the ability to androgenesis is manifested in larger pollen grains. A significant disadvantage of the anther culture is the uncertainty of the role of the somatic tissues of the rice anther during androgenesis and the possibility of the development of embryoids and callus both from microspores and from cells of somatic tissues of the anther wall and ligament. It is believed that the anther wall and connective tissue play an inducing role in the formation of androgenetic structures, however, there is no data on which specific anther tissues are involved in this. Studies of the ultrastructure of the tapetum (Batygina T.B., 1994) showed that the anther wall, in its specialization, integration and role in the development and differentiation of haploid structures and male gametophyte, is a unique sporophyte formation. This polyfunctional tissue directly adjoins the anther sporogenic tissue and ensures the passage of meiosis, the normal development of microspores, and the maturation of pollen grains. In general, the period of anther development, which is optimal for obtaining haploids, is species-specific in various flowering plants. The reasons may be the different type of formation of the anther wall (the number of layers, their origin, etc.), and hence the different structure of the anther wall at the same phase of development of the sporogenic tissue; different type of formation and structure of the tetrad of microspores $[9,10]$. Neoplasms obtained from somatic tissues are of no practical interest, since they repeat the genetic properties of the original parent. When developing regenerants in anther culture, plants originating from haploid structures (microspores) are important, since they are genetically unique and, if the set of chromosomes is doubled, have full homozygosity. Molecular characterization of DH lines from $F_{1}$ hybrids of rice (Oryza sativa L.) showed homozygosity for $99.84 \%$ of the total number of marker loci in 141 lines assessed, which suggests that the resulting plants originated from the pollen of $F_{1}$ generations donors $[11,12]$. There are publications describing the patterns leading to the formation of embryoids and / or callus from microspores or from somatic cells of anthers. So Tukavin G.B. (2007) notes that the formation of microspore cells or anther walls depends on the degree of differentiation of 
anther tissues, the stage of its development, and, accordingly, the stage of microspore development. It is reported that the transition from the gametophytic path of microspore development to the sporophytic pathway occurred during the cultivation of anthers containing microspores of the late mononuclear stage (before nuclear fission). At the same time, differentiated somatic tissues of the walls of cultivated anthers did not form callus, but the degree of tissue differentiation varied depending on the genotype [13]. For example, Kowalska U. et al. (2008) performed anatomical and cytological analyzes of the stages of embryogenesis and development of microspores in anther culture. Anthers were introduced into the culture, in which most of the microspores were at the optimal stage for the induction of androgenesis, that is, mononuclear. According to these studies, after one day of cultivation, several microspores increased and changed shape, on the second day of cultivation, microspores divided and formed structures of several cells, and after five days of cultivation in one anther it was possible to find an uncountable number of forming embryoids (from the first division of microspores to proembryo)[14]. It is possible that, as with any other stress, under in vitro cultivation conditions, the amount of endogenous hormones increases, as well as the relative content of some amino acids in the tissues of the anther wall, which can lead to the development of a larger number of viable competent microspores (P-pollen) and explain the effect of anther walls on the formation of embryo structures and regeneration of green plants. Thus, the study of the embryological aspects of the process of androgenesis in vitro is of particular interest, since this is one of the approaches that allow solving the acutely controversial issues associated with the problem of increasing the yield of embryoids in the isolated anthers culture. A systemic embryological approach to the theoretical and applied aspects of in vitro androgenesis has not been developed in the world literature. Meanwhile, it is absolutely necessary to use embryological information in this case. It is especially important to develop an approach to the anther as a complex integrated system, all elements of which (tissues of the anther nest wall, connective tissue and sporogenic tissue with its derivatives - microspores and pollen grains) develop interconnectedly and conjointly - both in vivo and in vitro [15].

The aim of this study was to study the development of microspores in rice anther culture in vitro; identification of cyto-embryological features of the formation of morphogenic structures in the androclinic callus of rice; obtaining doubled rice haploids (Oryza sativa L.) and accelerated development of valuable breeding material with desired properties.

\section{Material and methods}

In our study we used the method of isolated anthers culture. For cyto-histological studies and for development of genetically stabilized homozygous constant dihaploid lines (DH), explants of $\mathrm{F}_{1}$ and $\mathrm{BC}_{1}$ plants - rice generations obtained during hybridization and backcrossing between Russian and Chinese samples carrying blast resistance genes, were used. Donor plants for the selection of biomaterial (immature panicles) were grown in an open growing site and artificial climate chambers (ACC). The selection of panicles was based on cytological observations of the stage of microspore development using the EVOS XL Core Configured imaging system in transmitted light (bright field or phase contrast), lens range 1.25 - 100x, and then on morphological traits: panicle size, distance between the flag and the next leaf, color intensity of the flower and anther. Rice panicles were surface sterilized using $4 \%$ sodium hypochlorite $(\mathrm{NaClO})$ for 15 minutes and rinsed three times with sterile water. To inoculate the anthers, Blaydes' basic agar culture medium was used (Blaydes, 1966). The anthers were isolated under sterile conditions in a laminar box and evenly placed on the surface of the agar nutrient medium. Anthers were cultivated in a thermostat at a temperature of $25 \pm 20 \mathrm{C}$, relative humidity of $50.0 \%$ in the dark for $3-4$ weeks after inoculation. The response of the anthers to the processes of callus induction 
was monitored with the registration of data for each individual combination until the formation of callus mass. Calli were cultivated on Murashige and Scoogy medium (MS, 1962) with a photoperiod of 12 hours - day (5000 lux), 12 hours - night, until the appearance of green seedlings, which were transferred to a hormone-free MS medium for rooting.

Serial histological sections of anthers and calli were used to study the development of microspores and callus on nutrient media. The study and microphotography were performed using an MBI-6 microscope and an EVOS XL Core Configured imaging system. To count the chromosomes in the roots of rice regenerants and to determine the ploidy of test-tube plants, the method of squashed preparations was used. A macerated, acetocarmine-stained root was examined using an MBI-6 microscope, 40x and 70x objectives with water immersion (Pausheva, Z.P., 1974; Furst, G.G., 1979).

Haploid plants were transferred to the dihaploid level using the embryonic panicle method, in which panicles $0.5-0.7 \mathrm{~cm}$ in size were isolated in sterile boxing conditions and transferred to nutrient media to stimulate morphogenetic processes. The assessment of the genetic homogeneity of the obtained doubled rice haploids was carried out on the basis of genotyping of microsatellite DNA loci. When performing genotyping, multiplex PCR analysis was used. To perform DNA analysis, an ABIprism 3130 automatic genetic analyzer was used, which makes it possible to identify the difference in the size of the amplified fragments with an accuracy of one base pair.

\section{Results}

Accounting for the quantity and quality of androgenic calli was carried out for each sample. The analysis of the results showed that the variability in the callus formation trait is genetically determined, and therefore the induction of callusogenesis was noted not in all studied rice genotypes. When analyzing the average indicators of callusogenesis of the $F_{1}$ generation samples, the maximum result was shown by the samples Nautilus $x$ Liaojing 168 (21.65\%), Patriot x Liaoxing 21 (16.55\%), Novator x Liaojing 168 (11.48\%); the minimum - 0.31\% was noted for the sample VNIIR40 / 4-1 x Rongguang. Hybrid combintions Rapan x Rongguang, Patriot x Rongguang, Patriot x Liaojing 168, VNIIR 6753 x Rongguang, Nautilus x HY 11 did not have an androgenic response and did not induce processes of callusogenesis. When analyzing the average indicators of callusogenesis in samples of the $\mathrm{BC}_{1}$ generation, the maximum result was shown by samples (VNIIR40 / 4-1 x Fuguo) $\mathrm{x}$ VNIIR40 / 4-1 (17.85\%), (VNIIR 6753 x Rongguang) x VNIIR 6753 (13.0\%) and (Patriot $\mathrm{x}$ Liaojing 168) x Patriot (7.87\%). In the sample (VNIIR40 / 4-1 x Suijing 5) x VNIIR40 / $4-1$, induction of callusogenesis was not observed.

Variability in the regeneration trait, like callus formation, depended on the genotype. The best results in the implementation of form-building potencies and plant regeneration from calli were noted in samples $F_{1}$ Rapan $x$ Fuguo, $F_{1}$ Zlata $x$ Liaoxing 21, $B_{1}$ (VNIIR40 / 4-1 x Longing168) x VNIIR40 / 4-1 and BC 1 (VNIIR 6753 x Liaojing 168) $\mathrm{x}$ VNIIR 6753. This indicator for the listed genotypes was at the same level in the range of $2.96-3.89 \%\left(\mathrm{LSD}_{05}=1.205\right)$ (Table 1$)$.

The following samples were identified as responsive to the anther culture in vitro according to the complex of traits "callus formation / regeneration": F1 VNIIR40/4-1 x Longing3, F1 VNIIR40/4-1 x Tongyu 401, F1 Rapan x Fuguo, F1 Patriot x Liaoxing 21, F1 VNIIR 6753 x Suijing 5, F1 VNIIR 6753 x Suijing 5, F1 Nautilus x Liaojing 168, F1 Novator x Kendao 34, F1 Novator x Liaojing 168, F1 Ametist x Longgeng 31 and BC1 (VNIIR $6753 \times$ Liaojing 168) $x$ VNIIR 6753. In the rest of the studied genotypes, the dependence of the regeneration of green pollen seedlings on the frequency of callus formation was not revealed. 
Table 1. Callusogenesis and regeneration of $\mathrm{F}_{1}$ and $\mathrm{BC}_{1}$ - rice generations obtained in the course of hybridization and backcrossing between Chinese samples carrying blast resistance genes and Russian varieties.

\begin{tabular}{|c|c|c|c|}
\hline № & Combination name & Callusogenesis, \% & Regeneration, \% \\
\hline 1 & $\mathrm{~F}_{1}$ VNIIR40/4-1 x Rongguang & 0,31 & 0 \\
\hline 2 & F1 VNIIR40/4-1 x Fuguo & 0 & 0 \\
\hline 3 & F1 VNIIR40/4-1 x Longing3 & 3,26 & 27,9 \\
\hline 4 & $\mathrm{~F}_{1}$ VNIIR40/4-1 x Suijing 5 & 0,97 & 1,4 \\
\hline 5 & $F_{1}$ VNIIR40/4-1 x Tongyu 401 & 4,89 & 8,9 \\
\hline 6 & F1 VNIIR40/4-1 x Liaojing 168 & 3,57 & 1,5 \\
\hline 7 & $\mathrm{~F}_{1}$ VNIIR40/4-1 x Liaoxing 21 & 1,47 & 0 \\
\hline 8 & 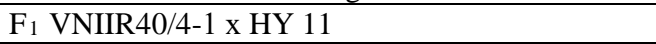 & 5,98 & 2,8 \\
\hline 9 & $\mathrm{~F}_{1}$ Rapan x Fuguo & 7,61 & 27,2 \\
\hline 10 & F1 Rapan x Kenddao 8 & 3,78 & 2,9 \\
\hline 11 & $\mathrm{~F}_{1}$ Rapan x Rongguang & 0 & 0 \\
\hline 12 & $\mathrm{~F}_{1}$ Patriot $\mathrm{x}$ Rongguang & 0 & 0 \\
\hline 13 & F1 Patriot x Liaojing 168 & 0 & 0 \\
\hline 14 & $\mathrm{~F}_{1}$ Patriot x Liaoxing 21 & 16,55 & 7,9 \\
\hline 15 & $\mathrm{~F}_{1}$ Patriot $\mathrm{x}$ HY 11 & 1,19 & 0 \\
\hline 16 & $F_{1}$ VNIIR $6753 \times$ Tianjing1 & 5,62 & 2,6 \\
\hline 17 & $F_{1}$ VNIIR $6753 \times$ Rongguang & 0 & 0 \\
\hline 18 & $F_{1}$ VNIIR 6753 x Suijing 5 & 2,42 & 15,9 \\
\hline 19 & $F_{1}$ VNIIR $6753 \times$ Liaojing 168 & 1,58 & 1,03 \\
\hline 20 & $\mathrm{~F}_{1}$ Zlata $\mathrm{x}$ Fuguo & 1,76 & 5,1 \\
\hline 21 & $\mathrm{~F}_{1}$ Zlata $\mathrm{x}$ Tongyu 401 & 4,10 & 4,0 \\
\hline 22 & $F_{1}$ Zlata x Liaojing 168 & 6,07 & 5,73 \\
\hline 23 & F1 Zlata x Liaoxing 21 & 5,47 & 11,1 \\
\hline 24 & $F_{1}$ Nautilus x Hejiang 20 & 7,1 & 11,7 \\
\hline 25 & $\mathrm{~F}_{1}$ Nautilus $\mathrm{x}$ Liaojing 168 & 21,65 & 16,8 \\
\hline 26 & $\mathrm{~F}_{1}$ Nautilus x HY 11 & 0,15 & 0 \\
\hline 27 & $F_{1}$ Kurazh x Suijing5 & 2,13 & 7,2 \\
\hline 28 & $F_{1}$ Novator x Longgeng 39 & 0,72 & 1,28 \\
\hline 29 & F1 Novator x Kendao 34 & 8,73 & 11,5 \\
\hline 30 & F1 Novator x Liaojing 168 & 11,48 & 16,7 \\
\hline 31 & $\mathrm{~F}_{1}$ Ametist $\mathrm{x}$ Longgeng 31 & 6,3 & 21,2 \\
\hline 32 & $F_{1}$ Zhemchug $\mathrm{x}$ Longgeng 31 & 9,72 & 4,1 \\
\hline 33 & $F_{1}$ Zhemchug x Longgeng 39 & 0,35 & 10,5 \\
\hline 34 & $\mathrm{~F}_{1}$ Zhemchug x Kendao 34 & 1,92 & 2,3 \\
\hline 35 & $\mathrm{~F}_{1}$ Khazar x Kendao 34 & 0,95 & 8,1 \\
\hline 36 & BC1 (VNIIR40/4-1 x Rongguang) x VNIIR40/4-1 & 3,56 & 0,73 \\
\hline 37 & BC1 $_{1}$ (VNIIR40/4-1 x Fuguo) x VNIIR40/4-1 & 17,85 & 1,08 \\
\hline 38 & 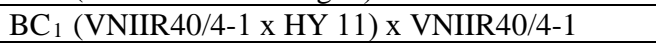 & 5,79 & 0 \\
\hline 39 & 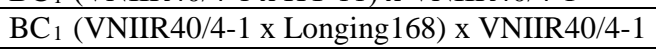 & 1,23 & 3,20 \\
\hline 40 & BC1 (VNIIR40/4-1 x Suijing 5) x VNIIR40/4-1 & 0 & 0 \\
\hline 41 & 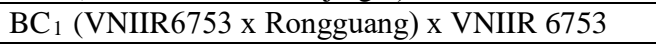 & 13,0 & 1,19 \\
\hline 42 & 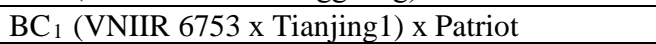 & 5,92 & 0 \\
\hline 43 & BC $_{1}$ (VNIIR 6753 x Liaoxing 21) x VNIIR 6753 & 7,20 & 0 \\
\hline 44 & BC $_{1}$ (VNIIR 6753 x Liaojing 168) x VNIIR 6753 & 3,61 & 2,96 \\
\hline 45 & BC $_{1}$ (Nautilus x Hejiang 20) x Nautilus & 1,25 & 0 \\
\hline 46 & BC1 (Rapan x Rongguang) x Rapan & 4,95 & 0,42 \\
\hline 47 & BC $_{1}$ (Patriot x Liaojing 168) $\mathrm{x}$ Patriot & 7,87 & 0,56 \\
\hline \multicolumn{2}{|c|}{ LSD 05} & 1,248 & 1,205 \\
\hline
\end{tabular}




\section{Discussion}

Cyto-histological studies of pollen sacs of the studied rice genotypes showed that already from the stage of microspore tetrads, degeneration (regeneration and death) of the anther wall cells took place, which was expressed in the destruction of the middle layer and lysis of the tapetum. By the time of anther inoculation on artificial nutrient media (late mononuclear and early binuclear stages of microspores), its wall consisted only of the epidermis and structureless fibrous endoderm. This indicated that during the cultivation of rice anthers in vitro, the cells of its binder and degenerative wall did not exhibit mitotic activity. They were not able to divide, form microcallus and induce somatic embryogenesis, since they did not have meristematic traits, therefore, they were not initial structures and served only for opening the anther. The only structures of which, under the influence of phytohormones, haploid androgenesis in rice is stimulated were microspores at the mononuclear or early binuclear stage of development. The use of anthers at an earlier (tetrad) and late (mature pollen grain) stages of development showed that they lack the morphogenetic potential for initiating sporophytic development in vitro. In a mature pollen grain, as a result of division, generative and vegetative cells were formed. The generative cell moved inside the vegetative one and, in a mature pollen grain, it was located next to its nucleus. The vacuole gradually disappeared, the cytoplasm was filled with reserve substances (proteins, lipids, starch granules).

\section{Development of rice microspores in anther culture in vitro}

During the cultivation of anthers containing microspores at the mononuclear and early binuclear stages of development, on nutrient media, only a few (competent) microspores were observed to have an abnormal development of nuclei. These deviations from the norm were noted from the initial stages. They were minimal after 5-10 days of exposure and were primary in the form of mild anaplasia. With further exposure of the anthers, a progression into the development of anomalies in microspores from a change in the nuclear-plasma ratio to mitotic division of individual cells was traced. Anaplasia in this period was expressed in a change in the structure and size of cell nuclei, which thickened and increased, there was a discrepancy in the protoplasm of the cytoplasmic mass with an enlarged nucleus. The nuclei lost their characteristic functions and acquired the ability for unlimited division and growth with the formation (on days 10 - 15 from the day of anther inoculation) of multicellular callus complexes (MC), which, increasing in size, ruptured the anther wall. Thus, the totipotency of microspores in vitro was realized during cultivation on nutrient media through abnormal fission of nuclei and initiation of callus formation processes.

At an early stage of development, callus aggregates located inside the anther nest were represented by a disorderly accumulation of cells that did not have the rudiments of embryonic structures (Figure 1). Calli appeared on the anther surface 35-40 days after inoculation. Differentiation of newly formed cells led to an increase in their mass and the formation of different types of tissue, as a result of which, in vitro-induced callus masses were characterized by a high degree of heterogeneity, even when they were obtained from the same donor genotypes. This was manifested in the morphological and structural diversity of calli. 


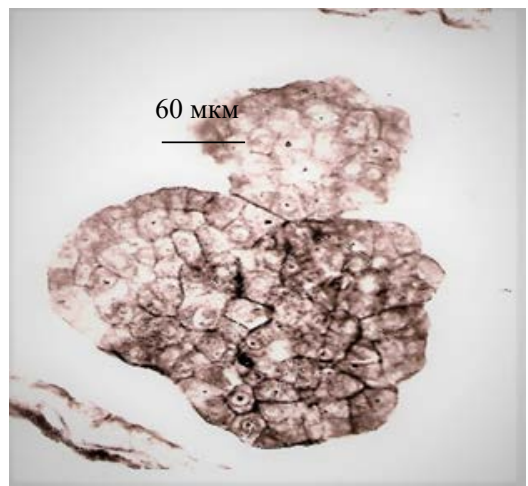

Fig. 1. Microcallus growth in the anther cavity of the $\mathrm{F}_{1}$ hybrid Rapan x Fuguo.

On histological sections of morphogenic rice callus, three types of cells were distinguished mainly - cells of the corona, meristem, and parenchyma. The crown is represented by loose tissue, consisting of small, vacuolated cells that limit the callus mass from atmospheric air. On the periphery of calli, foci of undifferentiated meristematic cells were formed in the form of protrusions. As a result of differentiation of these cells, growth points appeared, growth zones with large cells, a rounded large nucleus and a noticeable nucleolus, which were located in rows above the surface of the protrusions. In these zones, either embryo-like structures appeared - callus embryoids (secondary embryoids), or gemmas (buds) (Figure 2). The parenchyma lay deep in the callus and consisted of rounded (polygonal) cells of various sizes. Subsequently, vascular elements were laid in this tissue and vascular bundles were formed.

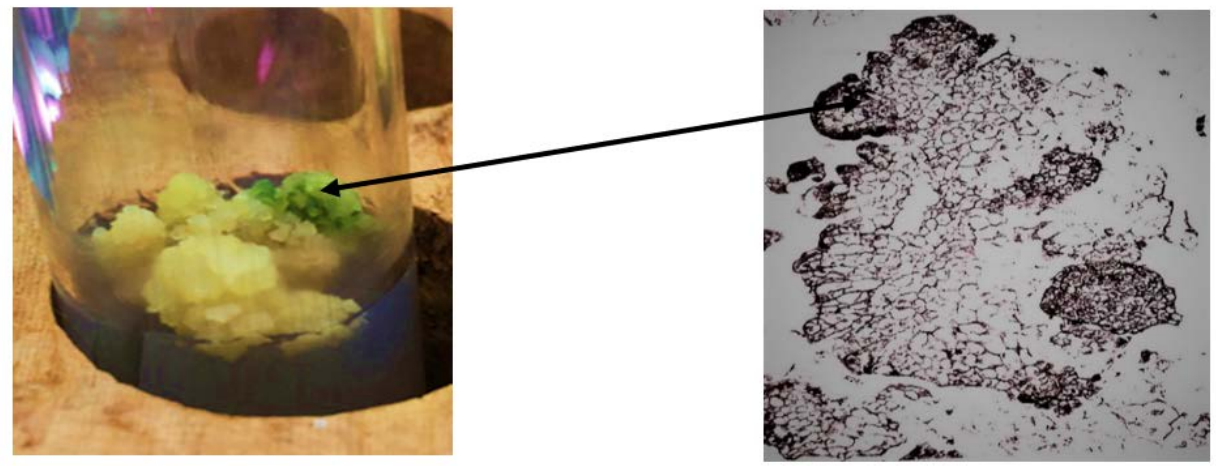

Fig. 2. Differentiation of rice callus meristem cells with the appearance of points and growth zones, F1 hybrid Rapan x Fuguo (left - morphogenic rice callus; right - histological section of morphogenic callus).

The multivariance of morphogenetic processes was observed, leading to the formation of different types of androgenic structures and pathways of organogenesis. During the cultivation of rice anthers, the spectrum of callus variability was quite wide; the formation of several morphotypes of calli was noted:

1) with meristematic foci, fine-grained, medium density, light shades, (morphogenic);

2) globular, medium density, white, light yellow (morphogenic);

3) nodular, light shades (morphogenic);

4) dense, fine-grained, white (morphogenic);

5) loose, wet, with vascular cords, white (rhizogenic);

6) granular, loose, with large cells, light brown (low ability to morphogenesis); 
7) soft, loose, wet, with large shapeless cells of various sizes, from light yellow to dark brown (non-morphogenic).

Calli morphology directly affected the regenerative capacity. The highest regenerative capacity was possessed by dense, white, fine-grained calli of a white or light yellow hue with white nodular inclusions. Their characteristics corresponded to "embryoidogenic callus". These callus tissues consisted of round, small cells with dense cytoplasm and a large nucleus, typical for meristem cells. Such calli were mainly formed in genotypes $\mathrm{F}_{1}$

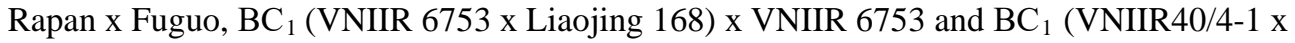
Longing168) x VNIIR40/4-1 (Figure 3).
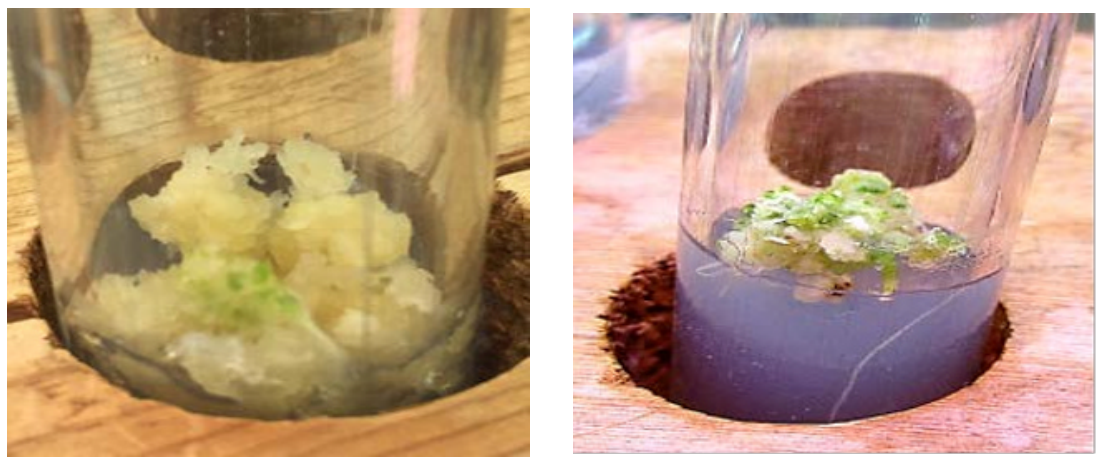

Fig. 3. Morphogenic rice callus of $F_{1}$ hybrid Rapan x Fuguo.

Round, white, light yellow, medium dense calli also had a high regenerative capacity. The cells of their tissues were somewhat larger, and the nuclei were less visible. Such callus aggregates were formed in samples $\mathrm{F}_{1}$ Novator $\mathrm{x}$ Kendao 34, $\mathrm{F}_{1}$ Zlata x Liaoxing 21, $\mathrm{F}_{1}$ VNIIR 40 / 4-1 x Tongyu 401, and F 1 Ametist x Longgeng 31.

The transfer of calli not only of different morphotypes, but also of different sizes to the regeneration medium revealed differences in their morphogenetic properties. The maximum yield of regenerants was obtained by young morphogenic 10-day-old calli 3-5 mm in size. There were few such callus aggregates; mainly, to stimulate morphogenesis, larger transplants $(5-10 \mathrm{~mm})$ were transferred to the regeneration medium. Cultivation of calli for 30 days provided a high yield of plants in relation to the number of original subcultured calli (Figure 4). Cultivation for more than 30 days reduced the morphogenetic potential of calli.

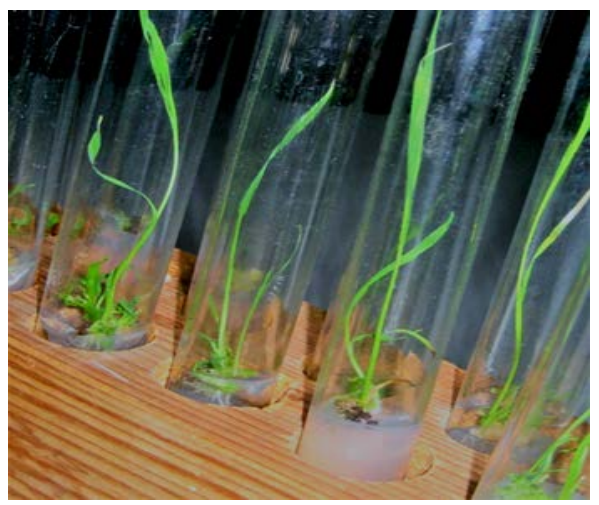

Fig. 4. Plant regeneration in rice Oryza sativa $L$ anther culture in vitro, $\mathrm{F}_{1}$ hybrid Rapan x Fuguo. 
The morphogenetic potencies of the studied rice samples were realized only through callus tissues by means of embryoidogenesis (formation of somatic embryoids of calli), gemmogenesis (formation of a bud), histogenesis (formation of tissues), or gemmorizogenesis (simultaneous formation of a bud and root). During the development of callus embryoids, the patterns noted in zygotic embryos were not always observed. In contrast to the strict dorso-ventral direction, a distortion in the direction of the stem and root of the callus embryoids was observed. Often there were such deviations as underdevelopment or absence of coleoptile, distortion of the shape of embryogenesis (two and three stem embryoids), often a multi-lobed embryonic root was formed, the directions of the stem and root could mutually intersect. In further development, a physiological gradient was observed - root-stem polarity.

The most active morphogenesis with the regeneration of viable green shoots was observed in 16 out of $35 \mathrm{~F}_{1}$ hybrids and in 6 out of $12 \mathrm{BC}_{1}$ generation genotypes introduced into anther culture in vitro. As a result, $\mathrm{R}_{1}$ progeny was obtained in the amount of 140 androgenic regenerant lines from $\mathrm{F}_{1}$ - generation hybrids and 40 lines from $\mathrm{BC}_{1}-$ generation (Table 2).

Table 2. Doubled haploid lines developed on the basis of $\mathrm{F}_{1}$ hybrids and $\mathrm{BC}_{1}$ generations.

\begin{tabular}{|c|c|c|}
\hline № & Combination name & $\begin{array}{c}\text { Androgenic } \mathrm{R}_{1} \text { lines, } \\
\text { pcs. }\end{array}$ \\
\hline 1 & $\mathrm{~F}_{1}$ Novator x Kendao 34 & 5 \\
\hline 2 & $\mathrm{~F}_{1}$ Novator x Longgeng 39 & 5 \\
\hline 3 & $\mathrm{~F}_{1}$ Novator $\mathrm{x}$ Liaojing 168 & 3 \\
\hline 4 & $\mathrm{~F}_{1}$ Rapan x Fuguo & 47 \\
\hline 5 & $\mathrm{~F}_{1}$ Ametist $\mathrm{x}$ Longgeng 31 & 11 \\
\hline 6 & $\mathrm{~F}_{1}$ VNIIR 40/4-1 x Tongyu 401 & 7 \\
\hline 7 & $\mathrm{~F}_{1}$ VNIIR40/4-1 x Longing3 & 3 \\
\hline 8 & $\mathrm{~F}_{1}$ VNIIR40/4-1 x Liaojing 168 & 4 \\
\hline 9 & $\mathrm{~F}_{1}$ VNIIR $6753 \times$ Tianjing1 & 5 \\
\hline 10 & $F_{1}$ VNIIR 6753 x Suijing5 & 4 \\
\hline 11 & $\mathrm{~F}_{1}$ Nautilus $\mathrm{x}$ Liaojing 168 & 14 \\
\hline 12 & $\mathrm{~F}_{1}$ Patriot $\mathrm{x}$ Liaoxing 21 & 5 \\
\hline 13 & $\mathrm{~F}_{1}$ Zlata $\mathrm{x}$ Liaoxing 21 & 18 \\
\hline 14 & $\mathrm{~F}_{1}$ Zlata $\mathrm{x}$ Liaojing 168 & 6 \\
\hline 15 & Zhamchug x Kendao 34 & 1 \\
\hline 16 & Khazar x Kendao 34 & 2 \\
\hline 17 & $\mathrm{BC}_{1}$ (Rapan x Rongguang) x Rapan & 1 \\
\hline 18 & $\mathrm{BC}_{1}$ (VNIIR 6753 x Rongguang) x VNIIR 6753 & 15 \\
\hline 19 & $\mathrm{BC}_{1}$ (VNIIR 6753 x Liaojing 168) x VNIIR 6753 & 9 \\
\hline 20 & $\mathrm{BC}_{1}$ (VNIIR 40/4-1 x Rongguang) x VNIIR 40/4-1 & 3 \\
\hline 21 & $\mathrm{BC}_{1}$ (VNIIR 40/4-1 x Fuguo) x VNIIR 40/4-1 & 3 \\
\hline 22 & $\mathrm{BC}_{1}$ (VNIIR 40/4-1 x Longing168) x VNIIR 40/4-1 & 9 \\
\hline & Total & 180 \\
\hline
\end{tabular}

Regenerant plants are rooted on liquid nutrient media and planted in vessels with soil for adaptation to ex vitro conditions. Currently, the plants are in different phases of the growing season: tillering, booting (going into the tube), heading and flowering, ripening. An assessment of the genetic homogeneity of the obtained rice doubled haploids was carried out on the basis of genotyping using 14 microsatellite DNA loci of doubled haploids obtained on the basis of $F_{1}$ hybrids. The homogeneity and stability of genotypes was noted 
in $97 \%$ of DH obtained, which indicates that these plants originated from donor pollen. It was noted that the majority of androgenic rice regenerants underwent spontaneous duplication of chromosomes (diploidization) through endomitosis of haploid callus cells, as a result of which stable doubled haploids from $\mathrm{F}_{1}$ and $\mathrm{BC}_{1}$ hybrids were obtained within one year. To double the number of chromosomes of haploid regenerants, we used the method of embryonic panicles $(0.5-0.7 \mathrm{~cm})$ and haploid tissues (nodes) with meristem cells, which were inoculated onto Blades medium with 2,4-D phytohormone at a concentration of $5.0 \mathrm{mg} / \mathrm{l}$. Preference is given to rudimentary panicles, because they are less susceptible to contamination. To induce shoot formation, callus aggregates were passaged on a Murashige and Skoog regeneration nutrient medium containing kinetin at a concentration of $5.0 \mathrm{mg} / \mathrm{L}$ and $\alpha$-NAA ( $\alpha$-naphthylacetic acid) at a concentration of $1.0 \mathrm{mg} / \mathrm{L}$.

Considering that doubled haploids are derived from plants obtained during hybridization and backcrossing between Russian accessions and Chinese samples carrying blast resistance genes, they will be tested for resistance to the Krasnodar population of rice blast pathogen in a growing area. Promising accessions - prototypes of future rice varieties with desired properties are planned to be submitted to variety testing, within the framework of which an accounting of the growing season, plant height, panicle mass, spikelet pubescence, field assessments of resistance to lodging and shedding, varietal uniformity of yield will be carried out. Biometrics will include panicle length and density, number of full and empty spikelets, percentage of sterile grain. The studied grain and milled rice quality traits include the mass of 1000 grains, filminess, vitreousity, grain size and index ( $/$ b), grain fracture, total milling yield and the head rice content. Based on the test results, a decision will be made on their further use in breeding programs.

\section{Conclusions}

The revealed relationships between the developmental stage of the male gametophyte and the morphological traits of rice panicle and anther made it possible to compare the data obtained and establish the phase of the functional activity of microspores during the transition from the gametophytic to the sporophytic (abnormal) path of development. Such microspores are at the mononuclear and early binuclear stages of development, have mitotic activity, and are able to divide with the formation of microcallus. In studies with rice anthers, processes were noted that occur only along the path of indirect androgenesis, with the formation of callus tissues and the development of secondary embryoids or buds (gems) in them, followed by organogenesis. The emergence of embryoids (androgenic embryos / globular pollen embryos) directly from microspores was not observed. The formation of viable rice regenerants was carried out in two ways: embryoidogenesis and / or hemmorizogenesis. In the embryoids, the coleoptile, the growth points of the shoot with the rudiments of the leaves, and the meristem of the embryonic root were laid. During hemorizogenesis in morphogenic types of calli, a bud was formed on the surface of the meristem focus, then a root was formed endogenously in the mass of callus, mainly in the basal part, at different distances and in different localization relative to the bud. Subsequently, between the gem and the root, the laying of vascular bundles took place, connecting them into a single plant organism - a regenerant.

The result of the study was an effective scheme for the accelerated development of homozygous rice breeding resources through the use of gamete cell technology, which has the following stages:

1. optimizing the conditions for growing donor plants;

2. determination of the optimal ontogenesis phase of donor plants for collecting explants (panicles); 
3. development of effective options for sterilization and temperature pretreatment of explants;

4. detailing the composition of artificial nutrient media in relation to rice genotypes for effective plant regeneration in vitro;

5. obtaining rice haploids and DH based on the studied genetic sources;

6. DNA analysis of genetic homogeneity (obtaining microsatellite profiles) and field tests of the morphological uniformity of the obtained androgenic lines with their subsequent reproduction to obtain seed progeny;

7. transfer of the obtained resources $\left(\mathrm{R}_{1}-\mathrm{R}_{2}\right)$ for inclusion into breeding schemes as prototype varieties with subsequent state registration (release).

\section{References}

1. Ohnoutkova L, Vlcko T, Ayalew M 2019 Methods in Molecular Biology 1900 37-52

2. Broughton S, Sidhu, P K, Davies P A 2014 Methods in Molecular Biology 1145 167189

3. Lantos C, Pauk J 2016 breeding Russian Journal of Genetics 52 794-801

4. Mishra R, Rao G J N, Ravi Rao N, Kaushal P 2015 Indica Rice Hybrids 22 6, http://dx.doi.org/10.1016/j.rsci.2015.07.002

5. Antonietta M 2011 Germanà Plant Cell, Tissue and Organ Culture (PCTOC) $104283-$ 300, https://doi.org/10.1007/s11240-010-9852-z

6. Lazaridou T, Sistanis I, Lithourgidis A, Ambrus H, Roupakias D Australian Journal of Crop Science 5 (3) 265-70

7. Alché J D, Castro A J, Solymoss M, Timar2 I, Barnabas B, Rodríguez-García M I 2000 Journal of Plant Physiology 156 146-155, https://doi.org/10.1016/S01761617(00)80299-0

8. Custódio L, Filomeno C M, Scientia A R 2005 Horticulturae 104 65-77 https://doi.org/10.1016/j.scienta.2004.08.001

9. Vlasov, V G, Savenko E G 2010 Rice growing 16 26-9

10. Savenko E G, Vlasov V G 2011 Rice growing 18 19-22

11. Epifanovich N V, Garkusha S V, Mukhina Zh M, Savenko E G et al. 2016 Book of reports, FSBSI «VNIIF», Bolshie Vyazemy 1 449-55

12. C. Lapitan V, D. Redoña E, Abe T, S. Brar D 2009 Field Crops Research 112 222-8, https://doi.org/10.1016/j.fcr.2009.03.008

13. Ssu-WeiHsu, Ming-CheLiu, Kuo-ChangZen, Co-ShineWang 2014 Plant Science 215216 124-33, https://doi.org/10.1016/j.plantsci.2013.11.006

14. Dyachuk T I, Khomyakova O V, Dugina T O 2010 Agricultural biology 5 61-5

15. Senadhira D, Zapata-Aria F J, Gregorio G B, Alejara M S, de la Cruz H C, Padolina T F, Galvez A M 2002 Field Crops Research 76 103-10 